\title{
Publisher Correction: DIY goes in vivo
}

Jim Kling

Correction to: Lab Animal, https://doi.org/10.1038/s41684-018-0066-z, published online 23 May 2018.

In the version of this Technology Feature originally published, André Maia Chagas' lab affiliation was omitted and the name of his institution, The Centre for Integrative Neuroscience, was incorrectly written. This has now been corrected. 